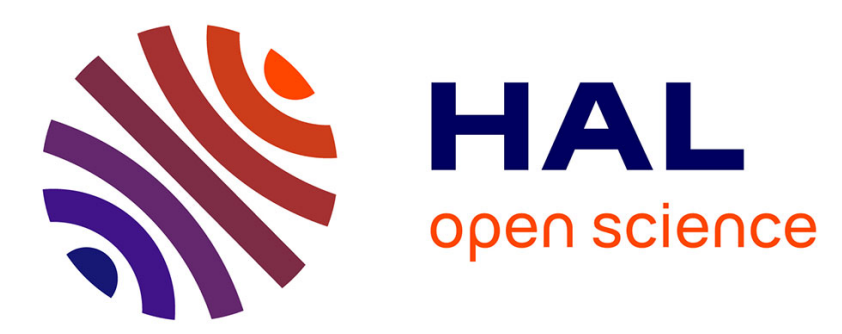

\title{
Ellipsoidal Enclosure Techniques for a Verified Simulation of Initial Value Problems for Ordinary Differential Equations
}

Andreas Rauh, Auguste Bourgois, Luc Jaulin, Julia Kersten

\section{To cite this version:}

Andreas Rauh, Auguste Bourgois, Luc Jaulin, Julia Kersten. Ellipsoidal Enclosure Techniques for a Verified Simulation of Initial Value Problems for Ordinary Differential Equations. 2021 International Conference on Control, Automation and Diagnosis (ICCAD), Nov 2021, Grenoble, France. pp.1-6, 10.1109/ICCAD52417.2021.9638755 . hal-03494430

HAL Id: hal-03494430

https://hal-ensta-bretagne.archives-ouvertes.fr/hal-03494430

Submitted on 19 Dec 2021

HAL is a multi-disciplinary open access archive for the deposit and dissemination of scientific research documents, whether they are published or not. The documents may come from teaching and research institutions in France or abroad, or from public or private research centers.
L'archive ouverte pluridisciplinaire HAL, est destinée au dépôt et à la diffusion de documents scientifiques de niveau recherche, publiés ou non, émanant des établissements d'enseignement et de recherche français ou étrangers, des laboratoires publics ou privés. 


\section{Ellipsoidal Enclosure Techniques for a Verified Simulation of Initial Value Problems for Ordinary Differential Equations}

\author{
Andreas Rauh, Auguste Bourgois, Luc Jaulin \\ Lab-STICC, ENSTA Bretagne \\ 29806 Brest, France \\ Andreas.Rauh@interval-methods.de, \\ Auguste.Bourgois@ensta-bretagne.org, \\ lucjaulin@gmail.com
}

\author{
Julia Kersten \\ Chair of Mechatronics \\ University of Rostock \\ D-18059 Rostock, Germany \\ Julia.Kersten@uni-rostock.de
}

\begin{abstract}
The verified simulation of initial value problems (IVPs) for ordinary differential equations (ODEs) with uncertain parameters is an up-to-date research topic and a basic building block for predictor-corrector type state estimators. Such state estimators are based on a two-stage procedure: First, the continuous-time state equations are evaluated up to the discrete time instant at which new measured data become available. Second, the forecasted state enclosures need to be refined by accounting for the information provided by the available sensors. In this paper, we focus on the first stage by presenting a novel ellipsoidal enclosure technique for continuous-time processes. It is based on thick ellipsoids and temporal Taylor series for a verified integration of ODEs that in combination allow for determining inner and outer bounds for the domains of reachable states. Comparisons with other set-valued integration techniques conclude this paper.
\end{abstract}

Index Terms-Ellipsoidal enclosure techniques; Interval analysis; Robustness analysis; Uncertain systems; Verified simulation of initial value problems

\section{INTRODUCTION}

The computation of guaranteed outer bounds for the sets of reachable states of IVPs for continuous-time ODEs has been addressed by many researchers over the recent years. State-ofthe-art solution techniques for systems with uncertain initial conditions and uncertain parameters make use of Taylor series expansions of the state trajectories over time or represent the solution sets by Taylor models which are high-order polynomials with additive interval-valued error bounds. In addition, techniques for a transformation of ODEs into a cooperative state-space representation were developed [1], [2]. In contrast to general solution techniques such as the solvers implemented in CAPD [3] or the INTLAB-based solver verifyode [4], which are commonly applied to compute the domains of reachable states directly for the complete parameter intervals and initial state domains, cooperative state-space representations aim at defining lower and upper bounding systems for the domains or reachable states which can be evaluated in a decoupled manner for so-called positive dynamic systems [5].
All of the aforementioned approaches have in common that they provide only outer bounds for the domains of reachable states. In such a way, they are not directly capable of quantifying the amount of overestimation due to multiple interval dependencies and the wrapping effect, cf. [6]. Sophisticated quality criteria such as those presented in [7] give indicators on the amount of pessimism in the computed state enclosures. However, they do not represent rigorous inner and outer bounds in a unified manner as proposed in [8], [9], where thick ellipsoids were introduced for the evaluation of nonlinear static functions and discrete-time dynamic systems.

In this paper, the definition of thick ellipsoids and their use for function evaluations and the specialized simulation of quasi-linear, parameter-dependent discrete-time systems are reviewed in Sec. II. In addition, a novel extension of this approach towards the verified integration of IVPs for ODEs is presented. The proposed methodology generalizes the Taylor series integration technique [10] to the use of thick ellipsoids. Sec. III provides - for the first time - a comparison of this new approach with the cooperativity-enforcing state-space transformation from [5] as well as the solvers CAPD [3] (using doubleton representations of the solution sets) and verifyode [4]. To perform this comparison, the simulation of the electric step-down converter circuit introduced in [5] is considered. Finally, conclusions and an outlook on future work are given in Sec. IV.

\section{Thick Ellipsoid Simulation Of Dynamic Systems}

\section{A. Definitions}

According to Fig. 1, thick ellipsoids are defined by outer and inner hulls that are parallel to each other. They are - during the evaluation of dynamic systems - computed in such a way that the interior of the inner bound is included with certainty in the domain of reachable states while there are certainly no reachable points outside the outer hull. The definition of thick ellipsoids and thick ellipsoid function extensions are recapitulated in the following on the basis of [8], [9]. 
Definition 2.1 (Thick ellipsoid): Define a thick ellipsoid $((\mathcal{E}))=((\mathcal{E}))(\boldsymbol{\mu}, \boldsymbol{\Gamma},[\rho ; \bar{\rho}])$, where $0 \leq \underline{\rho} \leq \bar{\rho}$, as a subset of the power set $\mathcal{P}\left(\overline{\mathbb{R}}^{n}\right)$ so that

$$
\begin{gathered}
((\mathcal{E}))=\left\{\mathcal{A} \in \mathcal{P}\left(\mathbb{R}^{n}\right) \mid \mathcal{E}^{\mathrm{I}} \subseteq \mathcal{A} \subseteq \mathcal{E}^{\mathrm{O}}\right\} \quad \text { with } \\
\mathcal{E}^{\mathrm{I}}=\left\{\mathbf{x} \in \mathbb{R}^{n} \mid(\mathbf{x}-\boldsymbol{\mu})^{T}(\underline{\rho} \boldsymbol{\Gamma})^{-T}(\underline{\rho} \boldsymbol{\Gamma})^{-1}(\mathbf{x}-\boldsymbol{\mu}) \leq 1\right\}, \\
\mathcal{E}^{\mathrm{O}=}\left\{\mathbf{x} \in \mathbb{R}^{n} \mid(\mathbf{x}-\boldsymbol{\mu})^{T}(\bar{\rho} \boldsymbol{\Gamma})^{-T}(\bar{\rho} \boldsymbol{\Gamma})^{-1}(\mathbf{x}-\boldsymbol{\mu}) \leq 1\right\} .
\end{gathered}
$$

Definition 2.2: (Thick ellipsoid binary operators and function extensions). A thick ellipsoid extension of the binary operators $\diamond \in\{+,-, \cdot, /, \cup, \cap\}$, where zero is assumed not to belong to the denominator for division, satisfies the relation

$$
\left\{\begin{array}{l}
\mathcal{A} \in((\mathcal{A})) \\
\mathcal{B} \in((\mathcal{B})) \\
\mathcal{C}=\mathcal{A} \diamond \mathcal{B}
\end{array} \quad \Longrightarrow \quad \mathcal{C} \in((\mathcal{A})) \diamond((\mathcal{B}))\right.
$$

The quantity $((\mathcal{C}))=((\mathcal{A})) \diamond((\mathcal{B}))$ is also a thick ellipsoid. Note that $((\mathcal{C}))$ is typically neither uniquely defined nor minimal with respect to its size. Moreover, $(\mathbf{f}))$ is a thick ellipsoid function extension of $\mathbf{f}: \mathbb{R}^{n} \mapsto \mathbb{R}^{m}$, if the property

$$
\left\{\begin{array}{l}
\mathcal{A} \in((\mathcal{A})) \\
\mathcal{B}=\mathbf{f}(\mathcal{A})
\end{array} \quad \Longrightarrow \quad \mathcal{B} \in((\mathcal{B}))=((\mathbf{f}))((\mathcal{A}))\right.
$$

is satisfied.
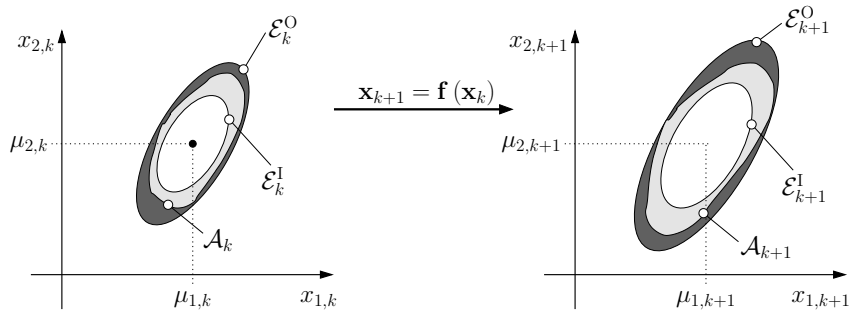

Fig. 1. Definition of a thick ellipsoid $((\mathcal{E}))_{k}$ enclosing the domain $\mathcal{A}_{k}$ and its mapping via the system model (5).

\section{B. Discrete-Time Case}

To evaluate discrete-time system models, a distinction between general nonlinear representations

$$
\mathbf{x}_{k+1}=\mathbf{f}\left(\mathbf{x}_{k}\right), \quad \mathbf{f}: \mathbb{R}^{n} \mapsto \mathbb{R}^{n}
$$

with differentiable right-hand sides $\mathbf{f}$ and quasi-linear ones

$\mathbf{x}_{k+1}=\mathbf{A}\left(\mathbf{x}_{k}, \mathbf{p}_{k}\right) \cdot \mathbf{x}_{k}+\boldsymbol{\delta}_{k}, \mathbf{A}\left(\mathbf{x}_{k}, \mathbf{p}_{k}\right) \in \mathbb{R}^{n \times n}, \boldsymbol{\delta}_{k} \in \mathbb{R}^{n}$

with the parameter vector $\mathbf{p}_{k}$ is made. In (5), uncertain parameters $\mathbf{p}_{k}$ are assumed to be included in the state vector $\mathbf{x}_{k}$ by means of discrete-time integrator disturbance models.

1) General Solution Approach: Consider the system model (5), where the state domain at the time instant $k$ is described by a thick ellipsoid $((\mathcal{E}))_{k}=((\mathcal{E}))_{k}\left(\boldsymbol{\mu}_{k}, \boldsymbol{\Gamma}_{k},\left[\underline{\rho}_{k} ; \bar{\rho}_{k}\right]\right)$. The parameters of the predicted thick ellipsoid $((\mathcal{E}))_{k+1}=((\mathcal{E}))_{k+1}\left(\boldsymbol{\mu}_{k+1}, \boldsymbol{\Gamma}_{k+1},\left[\underline{\rho}_{k+1} ; \bar{\rho}_{k+1}\right]\right)$ at the instant $k+1$, with its inner and outer bounds $\mathcal{E}_{k+1}^{\mathrm{I}}$ and $\mathcal{E}_{k+1}^{\mathrm{O}}$, are determined by Theorem 2.1 which was published in [9] together with a description in algorithmic form.

Theorem 2.1 ( [9] Thick ellipsoid enclosures): Given the thick ellipsoid state enclosure $((\mathcal{E}))_{k}$ at the time instant $k$ acc. to Def. 2.1 with a differentiable state equation (5), where

$$
\begin{gathered}
\mathbf{A}_{k}=\frac{\partial \mathbf{f}}{\partial \mathbf{x}_{k}}\left(\boldsymbol{\mu}_{k}\right) \quad \text { invertible } \\
((\mathcal{E}))_{k+1}=((\mathcal{E}))_{k+1}\left(\boldsymbol{\mu}_{k+1}, \boldsymbol{\Gamma}_{k+1},\left[\underline{\rho}_{k+1} ; \bar{\rho}_{k+1}\right]\right)
\end{gathered}
$$

is a thick ellipsoid enclosure of the set $\mathbf{f}\left(((\mathcal{E}))_{k}\right)$, if

$$
\boldsymbol{\mu}_{k+1}=\mathbf{f}\left(\boldsymbol{\mu}_{k}\right) \quad \text { and } \quad \boldsymbol{\Gamma}_{k+1}=\mathbf{A}_{k} \cdot \boldsymbol{\Gamma}_{k}
$$

as well as

$$
\underline{\rho}_{k+1}=\left(1-\rho_{\mathrm{I}, k}\right) \cdot \underline{\rho}_{k} \text { and } \bar{\rho}_{k+1}=\left(1+\rho_{\mathrm{O}, k}\right) \cdot \bar{\rho}_{k} .
$$

Here,

$$
\rho_{\mathrm{I}, k}=\max _{\left\|\tilde{\mathbf{x}}_{k}\right\| \leq 1}\left\|\tilde{\mathbf{b}}_{\mathrm{I}, k}\left(\tilde{\mathbf{x}}_{k}\right)\right\|
$$

is the maximum of the Euclidean norm of

$\tilde{\mathbf{b}}_{\mathrm{I}, k}\left(\tilde{\mathbf{x}}_{k}\right)=\underline{\rho}_{k}^{-1} \boldsymbol{\Gamma}_{k}^{-1} \mathbf{A}_{k}^{-1} \cdot\left(\mathbf{f}\left(\underline{\rho}_{k} \boldsymbol{\Gamma}_{k} \tilde{\mathbf{x}}_{k}+\boldsymbol{\mu}_{k}\right)-\mathbf{f}\left(\boldsymbol{\mu}_{k}\right)\right)-\tilde{\mathbf{x}}_{k}$

evaluated over the ball $\left\|\tilde{\mathbf{x}}_{k}\right\| \leq 1$ and

$$
\begin{gathered}
\rho_{\mathrm{O}, k}=\max _{\left\|\tilde{\mathbf{x}}_{k}\right\| \leq 1}\left\|\tilde{\mathbf{b}}_{\mathrm{O}, k}\left(\tilde{\mathbf{x}}_{k}\right)\right\| \\
\tilde{\mathbf{b}}_{\mathrm{O}, k}\left(\tilde{\mathbf{x}}_{k}\right)=\bar{\rho}_{k}^{-1} \boldsymbol{\Gamma}_{k}^{-1} \mathbf{A}_{k}^{-1} \cdot\left(\mathbf{f}\left(\bar{\rho}_{k} \boldsymbol{\Gamma}_{k} \tilde{\mathbf{x}}_{k}+\boldsymbol{\mu}_{k}\right)-\mathbf{f}\left(\boldsymbol{\mu}_{k}\right)\right)-\tilde{\mathbf{x}}_{k}
\end{gathered}
$$

2) Quasi-Linear System Models: For the special case of quasilinear systems (6), a discrete-time state prediction algorithm was derived in [8]. For simplicity, assume that $\boldsymbol{\delta}_{k}$ is a point vector. If this vector contains interval uncertainty, the quantities could be accounted for similar to Eqs. (11)-(14), treated as components of the state vector, or handled by the thick ellipsoid union operator derived in Sec. 3.3 of [8] to find thick ellipsoid bounds for the Minkowski sum of the corresponding sets. As visualized in Fig. 2, the evaluation is based on propagating an ellipsoid centered at the origin in parallel to an offset term in the form

$\mathbf{x}_{k+1}=\mathbf{A}\left(\mathbf{x}_{k}, \mathbf{p}_{k}\right) \cdot \check{\mathbf{x}}_{k}+\tilde{\mathbf{A}}_{k} \cdot \boldsymbol{\mu}_{k}+\left(\mathbf{A}\left(\mathbf{x}_{k}, \mathbf{p}_{k}\right)-\tilde{\mathbf{A}}_{k}\right) \cdot \boldsymbol{\mu}_{k}+\boldsymbol{\delta}_{k}$,

where

$$
\begin{aligned}
\mathbf{x}_{k} & \in((\mathcal{E}))_{k}\left(\boldsymbol{\mu}_{k}, \boldsymbol{\Gamma}_{k},\left[\underline{\rho}_{k} ; \bar{\rho}_{k}\right]\right), \\
\check{\mathbf{x}}_{k} & \in(\check{\mathcal{E}})_{k}\left(\mathbf{0}, \boldsymbol{\Gamma}_{k},\left[\underline{\rho}_{k} ; \bar{\rho}_{k}\right]\right), \\
\tilde{\mathbf{A}}_{k} & =\mathbf{A}\left(\boldsymbol{\mu}_{k}, \operatorname{mid}\left(\left[\mathbf{p}_{k}\right]\right)\right), \text { and } \\
\mathbf{p}_{k} & \in\left[\mathbf{p}_{k}\right]=\left[\underline{\mathbf{p}}_{k} ; \overline{\mathbf{p}}_{k}\right], \operatorname{mid}\left(\left[\mathbf{p}_{k}\right]\right)=\frac{1}{2} \cdot\left(\underline{\mathbf{p}}_{k}+\overline{\mathbf{p}}_{k}\right) .
\end{aligned}
$$

Using (15)-(19), a one time step evaluation of the quasi-linear model (6) is performed as follows [8]: 


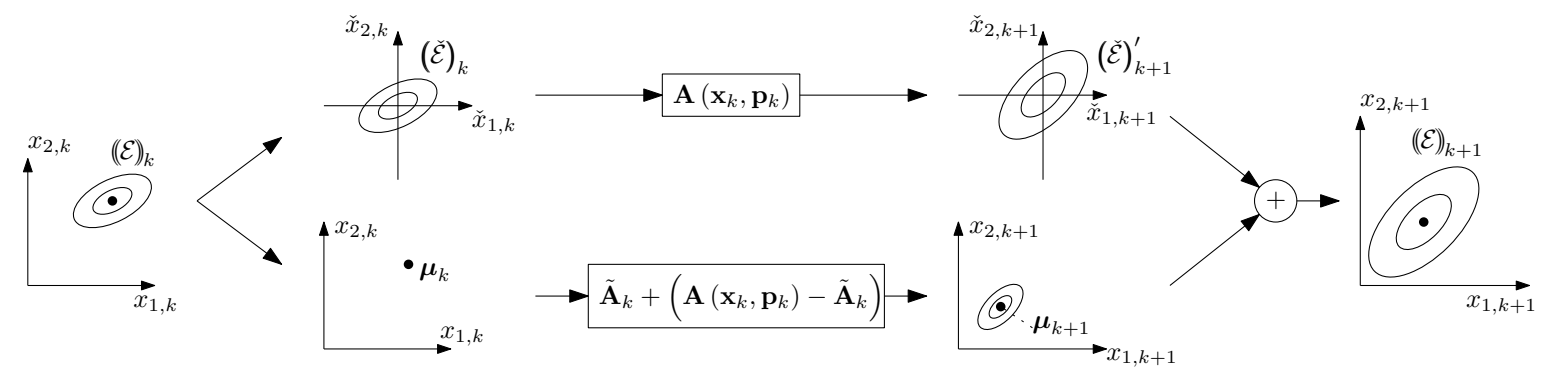

Fig. 2. Separation of the state equations according to (15)-(19) into the mapping of an origin-centered ellipsoid and the verified treatment of non-zero offset terms (visualized for the special case $\boldsymbol{\delta}_{k}=\mathbf{0}$ ).

1) Apply the mapping

$$
\check{\mathbf{x}}_{k+1}=\mathbf{A}\left(\mathbf{x}_{k}, \mathbf{p}_{k}\right) \cdot \check{\mathbf{x}}_{k},
$$

with $\mathbf{A}\left(\mathbf{x}_{k}, \mathbf{p}_{k}\right)$ evaluated for all $\mathbf{x}_{k} \in((\mathcal{E}))_{k}$ and $\mathbf{p}_{k} \in\left[\mathbf{p}_{k}\right]$, to the inner bound of $((\check{\mathcal{E}}))_{k}$ in (17). The shape matrix of the inner hull of the image set is given by

$$
\check{\mathbf{Q}}_{k+1}^{\mathrm{I}}=\alpha_{\mathrm{I}, k+1}^{2} \cdot \underline{\rho}_{k}^{2} \cdot \boldsymbol{\Gamma}_{k+1} \cdot \boldsymbol{\Gamma}_{k+1}^{T}, \mathbf{Q}_{k}=\underline{\rho}_{k}^{2} \cdot \boldsymbol{\Gamma}_{k} \cdot \boldsymbol{\Gamma}_{k}^{T},
$$

where $\alpha_{\mathrm{I}, k+1} \geq 0$ is the maximum value for which

$\mathcal{N}_{k+1}:=\boldsymbol{\Lambda}\left[\begin{array}{cc}\alpha_{\mathrm{I}, k+1}^{-2} \cdot \mathcal{R}_{k}^{-1} & \left(\tilde{\mathbf{A}}_{k}^{-1} \cdot \mathbf{A}\left(\mathbf{x}_{k}, \mathbf{p}_{k}\right)\right)^{-T} \\ \left(\tilde{\mathbf{A}}_{k}^{-1} \cdot \mathbf{A}\left(\mathbf{x}_{k}, \mathbf{p}_{k}\right)\right)^{-1} & \mathbf{Q}_{k}\end{array}\right] \begin{array}{r}\boldsymbol{\Lambda} \succeq 0 \\ (22)\end{array}$

is satisfied in terms of positive semi-definiteness with the typical choice $\mathcal{R}_{k}:=\rho_{k}^{2} \cdot \boldsymbol{\Gamma}_{k} \cdot \boldsymbol{\Gamma}_{k}^{T}$, cf. [8]. As a novel generalization of the procedure derived in [8], the symmetric positive definite preconditioning matrix $\boldsymbol{\Lambda}=\boldsymbol{\Lambda}^{T} \succ 0$ is introduced in (22) to optimize the ellipsoidal enclosures if the norms of $\left(\tilde{\mathbf{A}}_{k}^{-1} \cdot \mathbf{A}\left(\mathbf{x}_{k}, \mathbf{p}_{k}\right)\right)^{-T}$ and $\mathbf{Q}_{k}$ are significantly different, e.g., for very small state domains. In this paper, a block diagonal matrix $\boldsymbol{\Lambda}=\operatorname{blkdiag}\left(\beta \mathbf{I}, \beta^{-1} \mathbf{I}\right)$ with $\mathbf{I} \in \mathbb{R}^{n \times n}$ and the square root $\beta=\sqrt{\min \left\{\lambda_{i}\left(\mathbf{Q}_{k}\right)\right\}}$ of the smallest eigenvalue of $\mathbf{Q}_{k}$ is used. An optimization of $\boldsymbol{\Lambda}$ is a subject of future work.

2) Apply (20) to the outer bound of $(\check{\mathcal{E}})_{k}$ in (17). The shape matrix of the outer hull of the image set is given by

$$
\check{\mathbf{Q}}_{k+1}^{\mathrm{O}}=\alpha_{\mathrm{O}, k+1}^{2} \cdot \bar{\rho}_{k}^{2} \cdot \boldsymbol{\Gamma}_{k+1} \cdot \boldsymbol{\Gamma}_{k+1}^{T}, \mathbf{Q}_{k}=\bar{\rho}_{k}^{2} \cdot \boldsymbol{\Gamma}_{k} \cdot \boldsymbol{\Gamma}_{k}^{T},
$$

where $\alpha_{\mathrm{O}, k+1} \geq 0$ is the smallest value for which

$\mathcal{M}_{k+1}:=\boldsymbol{\Lambda}\left[\begin{array}{cr}-\mathbf{Q}_{k}^{-1} & \mathbf{A}^{T}\left(\mathbf{x}_{k}, \mathbf{p}_{k}\right) \cdot \tilde{\mathbf{A}}_{k}^{-T} \\ \tilde{\mathbf{A}}_{k}^{-1} \cdot \mathbf{A}\left(\mathbf{x}_{k}, \mathbf{p}_{k}\right) & -\alpha_{\mathrm{O}, k+1}^{2} \boldsymbol{\mathcal { R }}_{k}\end{array}\right] \mathbf{\Lambda} 0$

is satisfied for $\mathbf{x}_{k} \in(\mathcal{E})_{k}$ and $\mathbf{p}_{k} \in\left[\mathbf{p}_{k}\right], \boldsymbol{\mathcal { R }}_{k}:=\bar{\rho}_{k}^{2} \cdot \boldsymbol{\Gamma}_{k} \cdot \boldsymbol{\Gamma}_{k}^{T}$.

3) Compute interval bounds for the term

$$
\mathbf{b}_{k}=\left(\mathbf{A}\left(\mathbf{x}_{k}, \mathbf{p}_{k}\right)-\tilde{\mathbf{A}}_{k}\right) \cdot \boldsymbol{\mu}_{k} \in\left[\mathbf{b}_{k}\right]
$$

which account for a non-zero ellipsoid midpoint with $\mathbf{x}_{k}$, $\tilde{\mathbf{A}}_{k}$, and $\mathbf{p}_{k}$ defined according to (16), (18), and (19). Deflate the inner ellipsoid bound from (21) according to

$$
\begin{aligned}
\mathbf{Q}_{k+1}^{\mathrm{I}} & =\left(1-\rho_{\mathrm{I}, k+1}\right)^{2} \cdot \check{\mathbf{Q}}_{k+1}^{\mathrm{I}}, \\
\rho_{\mathrm{I}, k+1} & =\sup \left\{\left\|\alpha_{\mathrm{I}, k+1}^{-1} \cdot \underline{\rho}_{k}^{-1} \cdot \boldsymbol{\Gamma}_{k}^{-1} \cdot\left[\mathbf{b}_{k}\right]\right\|\right\}
\end{aligned}
$$

and inflate the outer bound in (23) with

$$
\begin{aligned}
\mathbf{Q}_{k+1}^{\mathrm{O}} & =\left(1+\rho_{\mathrm{O}, k+1}\right)^{2} \cdot \check{\mathbf{Q}}_{k+1}^{\mathrm{O}}, \\
\rho_{\mathrm{O}, k+1} & =\sup \left\{\left\|\alpha_{\mathrm{O}, k+1}^{-1} \cdot \bar{\rho}_{k}^{-1} \cdot \boldsymbol{\Gamma}_{k}^{-1} \cdot\left[\mathbf{b}_{k}\right]\right\|\right\} .
\end{aligned}
$$

For $\rho_{\mathrm{I}} \geq 1$, or if $\mathbf{A}\left(\mathbf{x}_{k}, \mathbf{p}_{k}\right)$ contains points at which it is not invertible, the inner bound becomes the empty set.

4) Compute the updated ellipsoid midpoint as

$$
\boldsymbol{\mu}_{k+1}=\tilde{\mathbf{A}}_{k} \cdot \boldsymbol{\mu}_{k}+\boldsymbol{\delta}_{k} .
$$

5) The thick ellipsoid at the time instant $k+1$ then becomes

$$
\mathbf{x}_{k+1} \in((\mathcal{E}))_{k+1}\left(\boldsymbol{\mu}_{k+1}, \boldsymbol{\Gamma}_{k+1},\left[\underline{\rho}_{k+1} ; \bar{\rho}_{k+1}\right]\right),
$$

where

$$
\begin{aligned}
\underline{\rho}_{k+1} & =\underline{\rho}_{k} \cdot \alpha_{\mathrm{I}, k+1} \cdot\left(1-\rho_{\mathrm{I}, k+1}\right), \\
\bar{\rho}_{k+1} & =\bar{\rho}_{k} \cdot \alpha_{\mathrm{O}, k+1} \cdot\left(1+\rho_{\mathrm{O}, k+1}\right), \text { and } \\
\boldsymbol{\Gamma}_{k+1} & =\tilde{\mathbf{A}}_{k} \cdot \boldsymbol{\Gamma}_{k} .
\end{aligned}
$$

Remark: Despite the novel extensions of the matrix inequalities (22) and (24) by the preconditioning $\Lambda$, these definiteness properties can be ensured according to [8] by a one-parameter optimization to determine $\alpha_{\mathrm{I}, k+1}$ and $\alpha_{\mathrm{O}, k+1}$. For a valid solution, all eigenvalues of $\mathcal{N}_{k+1}$ in (22) need to be nonnegative. Using the interval extension $\left[\mathcal{N}_{k+1}\right]$, bounds for all eigenvalues $\lambda_{i}, i \in\{1, \ldots, 2 n\}$, (cf. [11]) are obtained by

$$
\lambda_{i}\left(\operatorname{mid}\left(\left[\mathcal{N}_{\boldsymbol{k}+\mathbf{1}}\right]\right)\right)+\rho\left(\operatorname{rad}\left(\left[\mathcal{N}_{\boldsymbol{k}+\mathbf{1}}\right]\right)\right) \geq 0,
$$

where $\lambda_{i}\left(\operatorname{mid}\left(\left[\mathcal{N}_{k+1}\right]\right)\right)$ is the $i$-th eigenvalue of the midpoint matrix $\frac{1}{2} \cdot\left(\underline{\mathcal{N}}_{k+1}+\overline{\mathcal{N}}_{k+1}\right)$ and $\rho\left(\operatorname{rad}\left(\left[\mathcal{N}_{k+1}\right]\right)\right)$ the spectral radius of $\frac{1}{2} \cdot\left(\overline{\mathcal{N}}_{k+1}-\underline{\mathcal{N}}_{k+1}\right)$. Similarly, (24) is replaced by

$$
\lambda_{i}\left(\operatorname{mid}\left(\left[\mathcal{M}_{k+1}\right]\right)\right)+\rho\left(\operatorname{rad}\left(\left[\mathcal{M}_{k+1}\right]\right)\right) \leq 0 .
$$

\section{Continuous-Time Case}

To make the thick ellipsoid enclosure technique applicable to continuous-time systems

$$
\dot{\mathbf{x}}(t)=\mathbf{g}(\mathbf{x}(t), \mathbf{u}(t)),
$$

Eq. (35) is discretized on a given mesh $t_{k}$, where the (possibly interval-bounded) input vector $\mathbf{u}(t)$ is assumed to be piecewise 
constant for each $t_{k} \leq t<t_{k+1}=T_{k}+t_{k}$. Moreover, $\mathrm{g}$ is sufficiently often continuously differentiable, so that with $\mathbf{x}\left(t_{k}\right) \in\left[\mathbf{x}_{k}\right]$ an interval enclosure of the reachable states at $t_{k+1}$ can be determined by the temporal series expansion [10]

$$
\mathbf{x}\left(t_{k+1}\right) \in\left[\mathbf{x}_{k}\right]+\sum_{i=1}^{\nu} \frac{T_{k}^{i}}{i !} \cdot \mathbf{g}^{(i-1)}\left(\left[\mathbf{x}_{k}\right],\left[\mathbf{u}_{k}\right]\right)+\left[\mathbf{E}_{k}\right]
$$

where $\mathbf{g}^{(i-1)}$ are the solution's Taylor coefficients and

$$
\left[\mathbf{E}_{k}\right]:=\frac{T_{k}^{\nu+1}}{(\nu+1) !} \cdot \mathbf{g}^{(\nu)}\left(\left[\mathbf{B}_{\mathbf{x}, k}\right],\left[\mathbf{u}_{k}\right]\right)
$$

denotes an interval bound for the truncation error in which $\left[\mathbf{B}_{\mathbf{x}, k}\right]$ is a bounding box (computed with the help of a Picard iteration) of all possible states on the discretization interval $t \in\left[t_{k} ; t_{k+1}\right]$. For the special case of linear systems $\dot{\mathbf{x}}(t)=$ $\mathbf{A x}(t)+\mathbf{B u}(t)$ with piecewise constant inputs $\mathbf{u}(t)$, where $\mathbf{A}$ and $\mathbf{B}$ may contain uncertain but bounded and constant parameters, (36) and (37) turn into

$\mathbf{x}\left(t_{k+1}\right) \in \sum_{i=0}^{\nu} \frac{T_{k}^{i}}{i !} \cdot \mathbf{A}^{i} \cdot\left[\mathbf{x}_{k}\right]+\sum_{i=1}^{\nu} \frac{T_{k}^{i}}{i !} \cdot \mathbf{A}^{i-1} \cdot \mathbf{B} \cdot\left[\mathbf{u}_{k}\right]+\left[\mathbf{E}_{k}\right]$

and

$$
\left[\mathbf{E}_{k}\right]:=\frac{T_{k}^{\nu+1}}{(\nu+1) !} \cdot\left(\mathbf{A}^{\nu+1} \cdot\left[\mathbf{B}_{\mathbf{x}, k}\right]+\mathbf{A}^{\nu} \cdot \mathbf{B} \cdot\left[\mathbf{u}_{k}\right]\right) .
$$

During a thick ellipsoid evaluation of the system (35), the input interval $\left[\mathbf{u}_{k}\right]$ (assumed to be uncertain but temporally constant) is included in the state vector, so that the sum of the first two terms in (36) can be evaluated by means of the thick ellipsoid function evaluation of Sec. II-B1. Subsequently, a Picard iteration is carried out with the augmented state vector $\tilde{\mathbf{x}}_{k}=\left[\begin{array}{lll}\mathbf{B}_{\mathbf{x}, k}^{T} & t & \mathbf{x}_{k}^{T}\end{array}\right]^{T} \in \mathbb{R}^{2 n+1}$ using the calculus of thick ellipsoids. The equations for this iteration consist of $\boldsymbol{\Phi}\left(\tilde{\mathbf{x}}_{k}\right)=$ $\left[\mathbf{x}_{k}^{T}+t \cdot \mathbf{g}^{T}\left(\mathbf{B}_{\mathbf{x}, k}\right) \quad t \quad \mathbf{x}_{k}^{T}\right]^{T} \in \mathbb{R}^{2 n+1}$, where the iteration is initialized with an ellipsoid centered at the midpoint of the time interval $\left[t_{k} ; t_{k+1}\right]$ and the midpoint of $\left.(\mathcal{E})\right)_{k}$, where the initial shape matrix is given in the block diagonal form

$$
\tilde{\boldsymbol{\Gamma}}_{k}^{\langle 0\rangle} \cdot\left(\tilde{\boldsymbol{\Gamma}}_{k}^{\langle 0\rangle}\right)^{T}=\operatorname{blkdiag}\left(\bar{\rho}_{k}^{2} \boldsymbol{\Gamma}_{k} \boldsymbol{\Gamma}_{k}^{T},\left(\frac{T_{k}}{2}\right)^{2}, \bar{\rho}_{k}^{2} \boldsymbol{\Gamma}_{k} \boldsymbol{\Gamma}_{k}^{T}\right) .
$$

After convergence for some $\kappa>0$, the resulting augmented ellipsoid is projected onto the space of the first $n$ vector components by extracting the north western $n \times n$ block from the matrix $\tilde{\boldsymbol{\Gamma}}_{k}^{\langle\kappa\rangle} \cdot\left(\tilde{\boldsymbol{\Gamma}}_{k}^{\langle\kappa\rangle}\right)^{T}$. This projected ellipsoid forms the input for the evaluation of (37). In analogy to the error bounds (13), (14), the transformation matrix $\mathbf{T}_{\mathrm{O}}=\bar{\rho}_{k}^{-1} \boldsymbol{\Gamma}_{k}^{-1} \mathbf{A}_{k}^{-1}$ is multiplied from the left and $\mathbf{T}_{\mathrm{O}}^{T}$ from the right to its shape matrix. Finally, the resulting ellipsoid is enclosed by a ball whose radius is subtracted from the inner bound and added to the outer bound in analogy to (10) to obtain a verified enclosure of the errors $\mathbf{E}_{k}$ after also updating the ellipsoid midpoint.

Due to the linear state equations in the following application, the procedure of Sec. II-B2 is directly applied to the first sum in (38). The remaining two terms of this equation and formula (39) are directly evaluated in classical interval arithmetic. The resulting box is enclosed in a ball of radius $\rho_{\mathrm{E}, \mathrm{I}, k+1}$ (resp., $\left.\rho_{\mathrm{E}, \mathrm{O}, k+1}\right)$ in the coordinates $\underline{\rho}_{k+1} \boldsymbol{\Gamma}_{k+1} \cdot \mathbf{x}_{k+1}$ (resp. $\left.\bar{\rho}_{k+1} \boldsymbol{\Gamma}_{k+1} \cdot \mathbf{x}_{k+1}\right)$ so that the stretch parameters in (32) can be updated by the factors $\left(1-\rho_{\mathrm{E}, \mathrm{I}, k+1}\right)$ and $\left(1+\rho_{\mathrm{E}, \mathrm{O}, k+1}\right)$ in analogy to $\rho_{\mathrm{I}, k+1}$ and $\rho_{\mathrm{O}, k+1}$ in (32).

\section{ApPlication Scenario: SteP-Down CONVERTER}

As a benchmark for the proposed thick ellipsoid simulation technique and for a comparison with alternative approaches, the step-down converter circuit in Fig. 3 is considered [5]. It is assumed to be operated with a constant supply voltage $u_{0}=5 \mathrm{~V}$, the fundamental period $T=5 \mathrm{~ms}$, and the relative duty cycle length $\eta=0.6$. This duty cycle is implemented by periodically closing and opening the switch in Fig. 3 acc. to

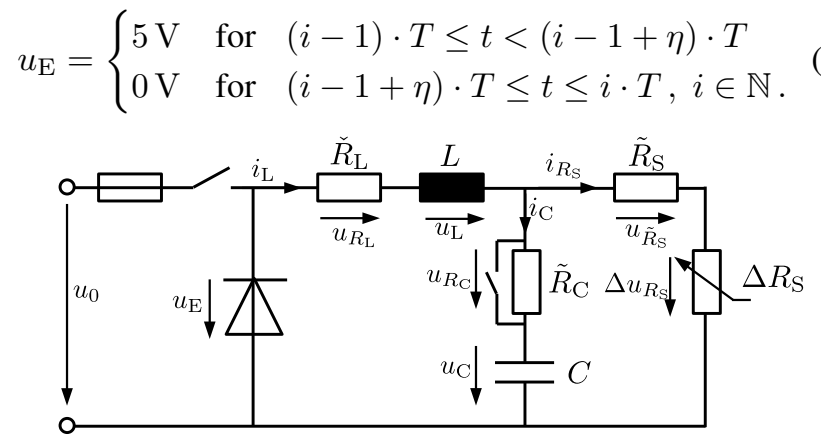

Fig. 3. Step-down converter.

According to [5], the circuit is described by the state equations

$$
\begin{aligned}
\dot{\mathbf{x}} & =\left[\begin{array}{cc}
-\frac{1}{L}\left(\check{R}_{\mathrm{L}}+\frac{R_{\mathrm{S}} R_{\mathrm{C}}}{R_{\mathrm{S}}+R_{\mathrm{C}}}\right) & \frac{1}{L}\left(\frac{R_{\mathrm{C}}}{R_{\mathrm{S}}+R_{\mathrm{C}}}-1\right) \\
\overline{R_{\mathrm{S}}} & -\frac{1}{C\left(R_{\mathrm{S}}+R_{\mathrm{C}}\right)}
\end{array}\right] \mathbf{x}+\left[\begin{array}{c}
\frac{1}{L} \\
0
\end{array}\right] \cdot u_{\mathrm{E}} \\
& =\mathbf{A}(\mathbf{p}) \cdot \mathbf{x}+\mathbf{b} \cdot u_{\mathrm{E}}
\end{aligned}
$$

with the inductor's current and the capacitor's voltage as state variables $\mathbf{x}=\left[\begin{array}{ll}x_{1} & x_{2}\end{array}\right]^{T}=\left[\begin{array}{ll}i_{\mathrm{L}} & u_{\mathrm{C}}\end{array}\right]^{T}$. For all simulations, the initial conditions are set to $\mathbf{x}(0)=\mathbf{0}$ with the system parameters $L=1 \mathrm{H}, \check{R}_{\mathrm{L}}=100 \Omega$ and $C=2 \mathrm{mF}$; $R_{\mathrm{C}} \in[0.1 ; 0.6] \Omega$ is composed of the internal resistance of the capacitor and the variably connectable element $\tilde{R}_{\mathrm{C}}$; $R_{\mathrm{S}} \in[0.1 ; 3] \Omega$ is an uncertain load consisting of the series connection $R_{\mathrm{S}}=\tilde{R}_{\mathrm{S}}+\Delta R_{\mathrm{S}}$ in which $\Delta R_{\mathrm{S}}$ can be activated and deactivated by semiconductor switches.

\section{A. Cooperativity-Enforcing Similarity Transformation}

In [5], it was shown that - for the considered parameter intervals - there exists no point-valued transformation $\mathbf{z}=\Theta^{-1} \cdot \mathbf{x}$ that turns (42) into the new state equations

$$
\dot{\mathbf{z}}=\hat{\mathbf{A}} \cdot \mathbf{z}+\mathbf{\Theta}^{-1} \cdot \mathbf{b} \cdot u_{\mathrm{E}} \quad \text { with } \quad \hat{\mathbf{A}} \in[\hat{\mathbf{A}}],
$$

in which the interval matrix $[\hat{\mathbf{A}}]$ is Metzler, i.e., it only contains off-diagonal entries with non-negative lower interval bounds. The interval matrix $[\hat{\mathbf{A}}]$ in (43) results from the transformation

$$
\hat{\mathbf{A}}(\mathbf{p})=\boldsymbol{\Theta}^{-1} \cdot \mathbf{A}(\mathbf{p}) \cdot \boldsymbol{\Theta} \in[\hat{\mathbf{A}}],
$$

where the dependence on the parameters $\mathbf{p}$ is accounted for by an entry-wise interval extension on the box $[\mathbf{p}]$. To obtain 
stable bounding trajectories - representing the underlying physics - it is necessary that all realizations of $[\hat{\mathbf{A}}]$ correspond to asymptotically stable dynamics (despite the wrapping effect of interval analysis [6] caused by the evaluation of (44)). Due to the lack of a single cooperativity-enforcing and stability-preserving change of coordinates, a multi-sectioning strategy for the parameter domain was developed in [5] which yields subboxes where on each of them the original system model can be transformed into asymptotically stable, cooperative state equations with the help of point-valued similarity transformations. The resulting parameter boxes are shown in Fig. 4 for two different parameterizations $N=10$ and $N=50$ (which denote the number of splittings performed in parallel).

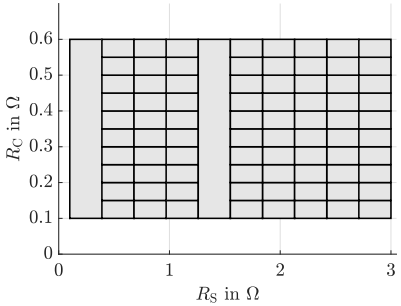

(a) Splitting with $N=10$.

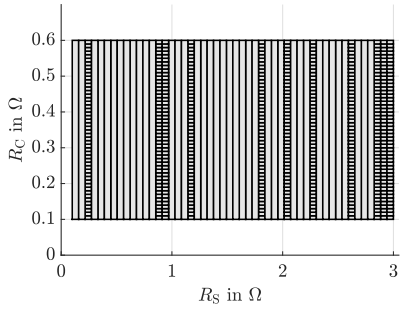

(b) Splitting with $N=50$.
Fig. 4. Partitioning of the parameter domain for two values $N$ in the multisectioning strategy of [5].

Performing simulations of the IVPs for each of these cooperative bounding systems, and transforming the convex interval hull back into the original coordinates, the state enclosures in Fig. 5 are obtained. Note, the implementation of the multisectioning strategy in [5] does not necessarily guarantee a decrease of the interval widths when arbitrarily increasing the splitting number $N$. This effect is visible in both subplots of Fig. 5, where the values $N=10$ and $N=50$ are compared. However, the computed bounds for the second state are much tighter than those resulting from a direct application of the solver verifyode in Sec. III-B. Even despite the splitting of the parameter domain, this approach also outperforms the following alternative with respect to computing times, cf. [5].

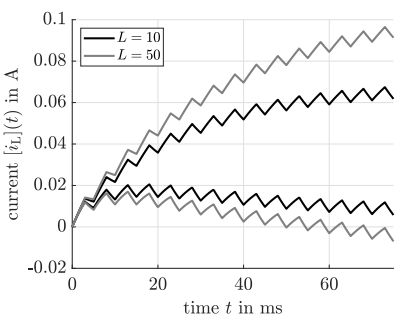

(a) State variable $x_{1}=i_{\mathrm{L}}$.

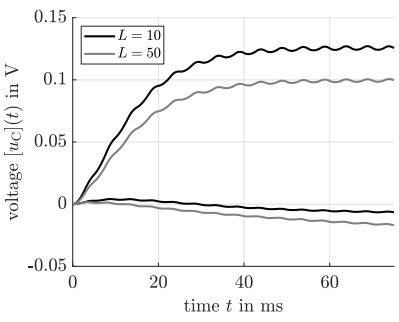

(b) State variable $x_{2}=u_{\mathrm{C}}$.
Fig. 5. State enclosures after a cooperativity-enforcing change of coordinates with the parameter box splitting according to Fig. 4.

\section{B. Taylor Model-Based Solution Approach}

For a simulation with the Taylor model-based ODE solver verifyode [4] from the MATLAB toolbox INTLAB V.12 [12], the uncertain parameters $\left[R_{\mathrm{C}}\right]$ and $\left[R_{\mathrm{S}}\right]$ are included in the state equations as interval variables specified by the datatype intval. For a successful simulation, the initial step size $\left(h_{0}=10^{-4}\right)$ and the minimum step size $\left(h_{\min }=10^{-6}\right)$ have to be set be sufficiently small. All further integrator options are set to the ones in the case study published in [5]:

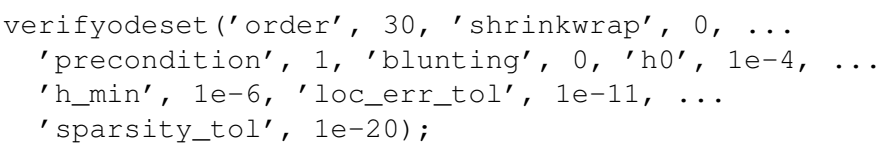

Using these settings, the comparison with the cooperativityenforcing state-space transformation from the previous subsection is performed. According to Fig. 6, the enclosures of the electric current $i_{\mathrm{L}}$ is tighter than the one of the previous approach, however, this solver fails to forecast the correct sign of $u_{\mathrm{C}}$. This makes the results of verifyode less applicable in practice because the indefinite sign of $u_{\mathrm{C}}$ does not allow to correctly determine the direction of power flow, i.e., to or from the load (where only the first makes sense from a physical point of view in the considered application).

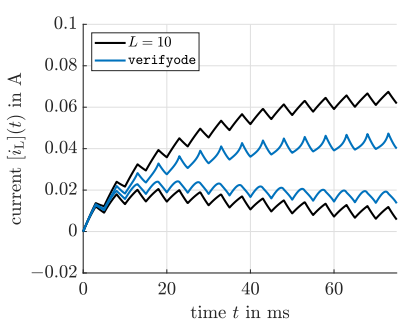

(a) State variable $x_{1}=i_{\mathrm{L}}$.

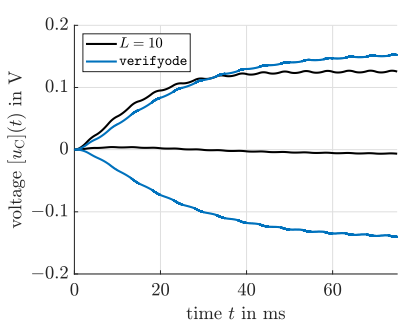

(b) State variable $x_{2}=u_{\mathrm{C}}$.
Fig. 6. Comparison between verifyode and the approach from Sec. III-A $(L=10)$.

\section{Taylor Expansion: CAPD as well as Box and (Thick) Ellipsoidal Enclosures}

As a final comparison, Fig. 7 shows that the solutions computed by CAPD using a doubleton representation diverge quickly if the same series expansion order of 30 is employed. In contrast, a direct evaluation of (36)-(37) by axis-aligned interval boxes provides the tightest bounds in this example, while the ellipsoidal counterpart is the best option for short integration time spans; however, it also breaks down (similarly to CAPD) within the second duty cycle. Note, all Taylor series-based evaluations were performed with the constant step size $T_{k}=10^{-4}$. The thick ellipsoidal enclosures have the property that their inner bounds are only non-empty if also the initial states have non-empty inner bounds. Therefore, the simulation is initialized at $t=0$ with a single point (i.e., empty inner bound), but re-initialized at the end of each blockwise defined input voltage interval in such a way that the inner bound is reset to the current outer bound. In such a way, the deviation between inner and outer bounds directly allows to quantify the worst-case pessimism of the solution over the respective time span as shown in Fig. 8(c), where the axis-aligned interval enclosures are depicted additionally. Due to the increasing width of the solutions over time, the inner bounds of the thick ellipsoids become empty in Fig. 8(d). Figs. 8(a) and 8(b) show that the ellipsoidal and CAPDbased enclosures start to widen initially in different directions. 
Hence, alternative specifications of the matrix $\boldsymbol{\mathcal { R }}_{k}$ in (21) can be used for tightening the enclosures in combination with the observer technique mentioned in the following conclusions.

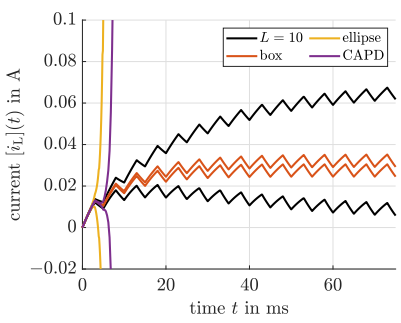

(a) State variable $x_{1}=i_{\mathrm{L}}$.

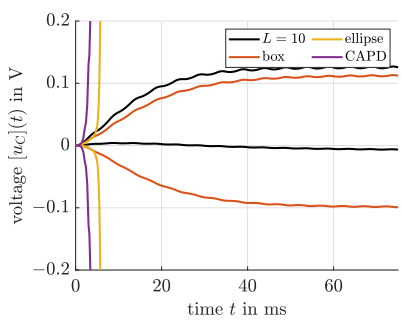

(b) State variable $x_{2}=u_{\mathrm{C}}$.
Fig. 7. Comparison between CAPD, the approach from Sec. III-A $(L=10)$ as well as Taylor series-based box and ellipsoid enclosures acc. to (36)-(39).

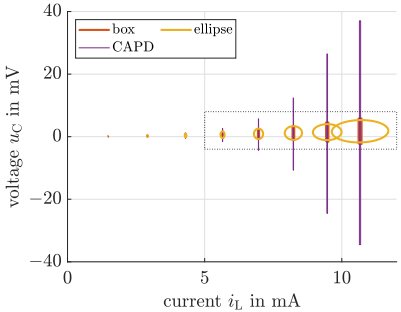

(a) Solution enclosures for $t_{k} \in$

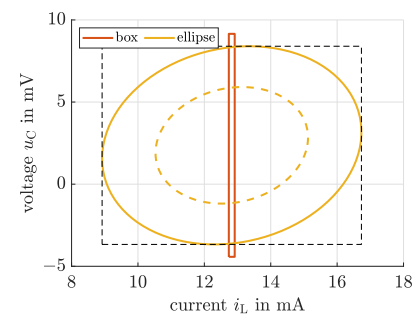

(c) Detailed view at $t_{k}=31 T_{k}$. $\left\{3 T_{k}, \ldots, 24 T_{k}\right\}$.

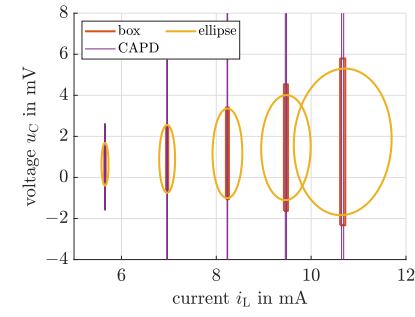

(b) Enlarged view of the dotted domain of Fig. 8(a).

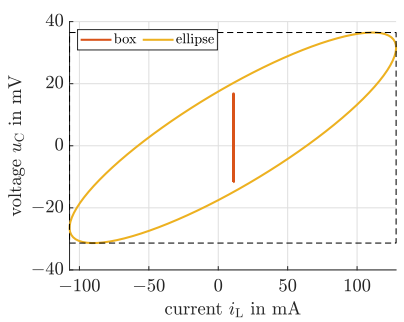

(d) Detailed view at $t_{k}=51 T_{k}$.
Fig. 8. Comparison between CAPD as well as Taylor series-based box and ellipsoid enclosures acc. to (36)-(39).

\section{CONCLUSiOnS AND Future WORK}

In this paper, a new extension of a thick ellipsoid simulation technique was presented for continuous-time systems. In previous work, this approach was only investigated for the discretetime case. The approach is capable of efficiently enclosing the state trajectories of dynamic systems, both from the inside and outside. Its bounds are comparable (or even tighter) than classical interval enclosures with comparable computational effort for sufficiently short time horizons.

Future work aims at validating the procedure for further nonlinear processes. There, it can be reasonable to intersect the results of various guaranteed enclosures (e.g. boxes and ellipsoids) at distinct points of time by the procedure published in [8]. According to Fig. 8(d), the ellipsoidal enclosures provide information about the correlation between various states. Hence, in contrast to axis-aligned boxes, an intersection with information (either simulation-based or measured in the case of predictor-corrector state estimators) for one of the state variables, also enhances the others. This is a big advantage over classical interval boxes, where such tightening can only be achieved by computationally expensive sub-paving approaches. Finally, the solution of differential inclusion problems will be investigated, which arise, e.g., if the inputs $\mathbf{u}$ of a dynamic system were specified by interval bounds, however, with the possibility for arbitrary variations in their interior. Then, higher-order Taylor series-based simulations are no longer applicable due to a lack of knowledge concerning interval bounds of the input derivatives [13]. Finally, possible extensions to fractional differential equations will be considered [14].

\section{REFERENCES}

[1] D. Angeli and E. Sontag, "Monotone Control Systems," IEEE Transactions on Automatic Control, vol. 48, no. 10, pp. 1684-1698, 2003.

[2] D. Efimov, T. Raïssi, S. Chebotarev, and A. Zolghadri, "Interval State Observer for Nonlinear Time Varying Systems," Automatica, vol. 49, no. 1, pp. 200-205, 2013.

[3] T. Kapela, M. Mrozek, D. Wilczak, and P. Zgliczynski, “CAPD::DynSys: A Flexible C++ Toolbox for Rigorous Numerical Analysis of Dynamical Systems," Communications in Nonlinear Science and Numerical Simulation, p. 105578, 112020.

[4] F. Bünger, “A Taylor Model Toolbox for Solving ODEs Implemented in MATLAB/INTLAB," Journal of Computational and Applied Mathematics, vol. 368, p. 112511, 2020.

[5] A. Rauh and J. Kersten, "Transformation of Uncertain Linear Systems with Real Eigenvalues into Cooperative Form: The Case of Constant and Time-Varying Bounded Parameters," Algorithms, vol. 14, no. 3, 2021. [Online]. Available: https://www.mdpi.com/1999-4893/14/3/85

[6] L. Jaulin, M. Kieffer, O. Didrit, and É. Walter, Applied Interval Analysis. London: Springer-Verlag, 2001.

[7] E. Auer and A. Rauh, "Toward definition of systematic criteria for the comparison of verified solvers for initial value problems," in Parallel Processing and Applied Mathematics, R. Wyrzykowski, J. Dongarra, K. Karczewski, and J. Wasniewski, Eds. Berlin, Heidelberg: Springer Berlin Heidelberg, 2010, pp. 408-417.

[8] A. Rauh, A. Bourgois, and L. Jaulin, "Union and Intersection Operators for Thick Ellipsoid State Enclosures: Application to Bounded-Error Discrete-Time State Observer Design," Algorithms, vol. 14, no. 3, 2021. [Online]. Available: https://www.mdpi.com/1999-4893/14/3/88

[9] A. Rauh and L. Jaulin, "A Novel Thick Ellipsoid Approach for Verified Outer and Inner State Enclosures of Discrete-Time Dynamic Systems," in Proc. of 19th IFAC Symposium System Identification: Learning Models for Decision and Control, online, 2021.

[10] N. S. Nedialkov, "Computing Rigorous Bounds on the Solution of an Initial Value Problem for an Ordinary Differential Equation," Ph.D. dissertation, Graduate Department of Computer Science, University of Toronto, 1999.

[11] J. Rohn, "Positive Definiteness and Stability of Interval Matrices," SIAM Journal on Matrix Analysis and Applications, vol. 15, no. 1, pp. 175184, 1994.

[12] S. Rump, "INTLAB - INTerval LABoratory," in Developments in Reliable Computing, T. Csendes, Ed. Kluver Academic Publishers, 1999, pp. 77-104

[13] S. Rohou and L. Jaulin, "Exact Bounded-Error Continuous-Time Linear State Estimator," Systems \& Control Letters, vol. 153, p. 104951, 2021.

[14] A. Rauh and L. Jaulin, "Novel Techniques for a Verified Simulation of Fractional-Order Differential Equations," Fractal and Fractional, vol. 5, no. 1, 2021. [Online]. Available: https://www.mdpi.com/2504$3110 / 5 / 1 / 17$ 